\title{
Delirium e fatores associados em unidades de terapia intensiva: estudo piloto de coorte
}

\author{
Delirium and associated factors in \\ intensive care units: cohort pilot study
}

\section{Mariana Santos Lago' ${ }^{1}$ Tássia Nery Faustino ${ }^{2}$ (1) Magno Conceição das Mercês ${ }^{3}$ (1) Douglas de Souza e Silva ${ }^{4}$ (i) Larissa Simões da Cruz Pessoa 5 (1) Muriel Trindade Santos Oliveira ${ }^{6}$ (1)}

${ }^{1}$ Autora para correspondência. Universidade do Estado da Bahia. Salvador, Bahia, Brasil. marilago91@gmail.com 2,3,6 Universidade do Estado da Bahia (Salvador). Bahia, Brasil. tassiafaustino@yahoo.com.br, magnomerces@hotmail.com, oliveira.muriel6@gmail.com 4,5Universidade Federal da Bahia (Salvador). Bahia, Brasil. douglasssgbi@hotmail.com, larissacruz7@outlook.com

RESUMO | OBJETIVOS: Detectar a incidência de delirium e os fatores associados à sua ocorrência em unidades de terapia intensiva (UTIS). MÉTODO: Estudo piloto de coorte efetuado em três UTIs. A amostra foi composta de pacientes adultos clínicos e cirúrgicos, com tempo de internamento superior a 24 horas. O delirium foi monitorizado através do Confusion Assessment Method for the Intensive Care Unit. Utilizaram-se os testes Quiquadrado de Pearson e Exato de Fisher para comparação entre os grupos, considerando-se como estatisticamente significantes os valores de $p<0,05$. Os resultados também foram apresentados através do Risco Relativo. RESULTADOS: A amostra foi constituída por 59 pacientes, sendo que destes 33,9\% $(n=20)$ cursaram com delirium, com predominância do espectro hipoativo, com $50 \%$ dos casos $(n=10)$. Ressalta-se que $90 \%$ dos pacientes apresentaram o início dos episódios nas primeiras 24 horas de internamento e a média de duração dos episódios foi de aproximadamente 02 dias. Dentre as variáveis pesquisadas, houve uma associação positiva e estatisticamente significante entre o uso de contenção física e ocorrência da disfunção ( $R R=3,10 ; p=0,01)$. CONCLUSÃO: A incidência de delirium na amostra foi elevada, predominando o subtipo hipoativo. $\mathrm{O}$ uso de contenção física elevou em três vezes o risco de desenvolvimento do delirium.

DESCRITORES: Delirium. Adulto. Equipe de enfermagem. Cuidados críticos. Unidades de Cuidados Intensivos.

\begin{abstract}
OBJECTIVES: To detect the incidence of the delirium and the associated facors with its occurrence in intensive care units (ICUs). METHOD: Cohort pilot study conducted in three ICUs. The sample consisted of adult clinical and surgical patients with hospitalization time greater than 24 hours. Delirium was monitored by the Confusion Assessment Method for the Intensive Care Unit. Pearson's chi-square and Fisher's exact tests were used for comparison between groups, considering $p<0.05$ as statistically significant. Results were also presented through Relative Risk. RESULTS: The sample consisted of 59 patients, of which $33.9 \%(n=20)$ had delirium, with predominance of the hypoactive spectrum, with $50 \%$ of cases $(n=10)$. It stand out that $90 \%$ of patients had onset of episodes within the first 24 hours of hospitalization and the average duration of episodes was approximately 02 days. Among the researched variables, there was a positive and statistically significant association between the use of physical restraint and occurrence of dysfunction ( $R R=3.10$; $p=0.01)$. CONCLUSION: The incidence of delirium in the sample was high, predominating the hypoactive subtype. The use of physical restraint increased by three times the risk of developing delirium.
\end{abstract}

DESCRIPTORS: Delirium. Adult. Nursing team. Critical Care. Intensive Care Units. 


\section{Introdução}

O delirium pode ser conceituado como uma disfunção cerebral que apresenta como característica principal a perturbação do nível de atenção, consciência e cognição que acontece de forma aguda e flutuante, acarretando prejuízos cognitivos que não podem ser explicados por algum transtorno neurocognitivo prévio ou em curso'. De forma complementar, o delirium pode ser classificado de acordo com o nível de atividade motora entre os tipos hipoativo, hiperativo ou misto ${ }^{1,2}$. No ambiente da unidade de terapia intensiva, estima-se uma prevalência de delirium de $31 \%$, sendo que o subtipo mais encontrado é o hipoativo, estando relacionado a indivíduos com maior gravidade da doença, seguido do espectro misto ${ }^{2}$.

Associadamente, tem-se comprovado que o delirium está relacionado a desfechos clínicos negativos, como aumento da mortalidade, tempo de internamento e custos hospitalares e elevado índice de comprometimento cognitivo após alta hospitalar ${ }^{3-5}$. Entretanto, não é apenas a ocorrência do delirium que tem preocupado os profissionais de saúde, mas também a sua duração, visto que pesquisas informam que quanto maior o número de dias com a disfunção, maior será a perda cognitiva do paciente ${ }^{3}$.

Devido ao subdiagnóstico do delirium através de uma avaliação clínica usual, indica-se a utilização de escalas para a sua detecção e monitorização contínua, sendo que existem várias instrumentos validados para o diagnóstico dessa disfunção em pacientes críticos, contudo recomenda-se o uso da Confusion Assessment Method for the ICU (CAM-ICU) e o Intensive Care Delirium Screening Checklist (ICDSC) devido às mesmas apresentarem elevada sensibilidade e especificidade, além de facilidade e rapidez de aplicação na prática clínica 5 .
Aliado ao diagnóstico precoce do delirium, a prevenção e manejo dos fatores de risco para a síndrome são imprescindíveis. Sabe-se que existem diversos fatores de risco associados ao delirium, são estes classificados em: 1) Fatores predisponentes, considerados como não modificáveis, são eles: idade avançada, coma prévio, demência, alcoolismo, hipertensão, depressão, alta gravidade da doença no momento da admissão na UTI, dentre outros; e 2) Fatores precipitantes, considerados como modificáveis, são eles: uso de fármacos com ação anticolinérgica, imobilização, transfusão sanguínea, privação sensorial, distúrbios do sono, entre outros ${ }^{5-7}$.

Apesar da magnitude dessa disfunção e da sua forte associação com piores desfechos para o paciente crítico, verificou-se através de uma pesquisa eletrônica nas bases de dados Scientific Electronic Library Online (SCIELO), Literatura Latino-Americana e do Caribe em Ciências da Saúde (LILACS) e Medical Literature Analysis and Retrieval System Online (MEDLINE), utilizando o cruzamento dos descritores "delirium", "adulto", "equipe de enfermagem" e "cuidados críticos" que dados referentes à temática no cenário Brasileiro são escassos e não foram encontradas publicações referentes à incidência da disfunção em UTIs. Além disso, faz-se necessário identificar os fatores associados ao delirium para a implementação precoce de medidas preventivas.

Face ao exposto, esse artigo objetivou detectar a incidência de delirium e os fatores associados à sua ocorrência em unidades de terapia intensiva de um hospital público.

\section{Método}

Trata-se de um estudo piloto de coorte prospectivo efetuado em três unidades de terapia intensiva de um hospital público e de grande porte. A partir dos dados fornecidos por esse piloto (incidência de delirium), pretende-se efetuar um estudo com amostra probabilística para avaliar a efetividade de intervenções cognitivas na redução da duração do delirium nos pacientes internados nessas UTIs. 
A amostra não probabilística de conveniência foi constituída por pacientes clínicos, neurológicos e cirúrgicos, maiores de 18 anos e com tempo de internamento na UTI superior a 24 horas que estiveram internados no locus do estudo no período de novembro de 2018 a janeiro de 2019. Foram excluídos os pacientes com pontuação na Richmond AgitationSedation Scale (RASS) $\leq-3$, admitidos em delirium, com afasia receptiva, com grave deficiência auditiva que dificultasse a comunicação e com transtornos psiquiátricos que ocasionassem alucinações.

O delirium foi monitorizado através do CAM-ICU, já traduzido e validado para o português, aplicado diariamente no turno vespertino durante todo o internamento do paciente na UTI por três pesquisadores (dois enfermeiros residentes em Terapia Intensiva e uma graduanda em Enfermagem) e em regime de escala, após capacitação teórica e treinamento in loco para aplicação do instrumento.

Determinou-se como variável dependente a ocorrência de delirium (sim/não) e como variáveis independentes (sim/não): diagnóstico médico de hipertensão e alcoolismo, uso de ventilação mecânica, sedação, anticolinérgicos, opióides, contenção física e transfusão sanguínea.

A escala CAM-ICU avalia 4 características do paciente, sendo elas: 1) Alteração aguda ou flutuação do estado mental; 2) Desatenção; 3) Pensamento desorganizado; e 4) Nível de consciência alterado no momento da avaliação. Para que o diagnóstico do delirium seja positivo é necessário a presença obrigatória dos itens 1 e 2 associados à presença do item 3 e/ou $4^{8}$. A classificação do subtipo motor do delirium baseou-se na pontuação alcançada na escala de RASS no momento em que o resultado do CAM-ICU foi positivo: RASS -1 ou -2 como delirium hipoativo; RASS +1 a +4 como delirium hiperativo; RASS 0 ou variando no decorrer dos dias entre os subtipos anteriores, como delirium misto. A duração do delirium foi determinada pelo número de dias de CAM-ICU positivo da admissão até a alta do participante da UTI.
Os dados sociodemográficos e clínicos dos participantes (idade, sexo, SAPS 3, dias de internamento e diagnóstico de admissão na UTI) e as variáveis independentes investigadas foram coletados através dos prontuários. Não houve perdas de seguimento dos participantes durante o estudo.

Os dados coletados foram digitados no programa computacional Microsoft ${ }^{\circledR}$ Excel e posteriormente analisados pelo programa Statitical Package for the Social Science (SPSS) versão $22.0 \AA$. Os resultados foram apresentados através da estatística descritiva por meio de frequências absolutas e relativas e por médias e desvios-padrões de acordo as variáveis categóricas ou quantitativas, respectivamente.

Para a comparação dos grupos, foi empregado o teste Qui-quadrado de Pearson e o teste Exato de Fisher, considerando-se o valor $p<0,05$ para estabelecer diferenças estatísticas significativas. Os resultados também foram apresentados através do Risco Relativo (RR) com Intervalo de Confiança (IC) de 95\%.

Esse estudo foi aprovado pelo Comitê de Ética (CAAE 97131118900000057) e foram seguidas todas as recomendações necessárias para a pesquisa com seres humanos determinadas na Resolução n 466 de 12 dezembro de $2012^{9}$. O Termo de Consentimento Livre e Esclarecido foi obtido de todos os participantes do estudo, após esclarecimento acerca dos objetivos e operacionalização da pesquisa, assim como os riscos e benefícios envolvidos.

\section{Resultados}

A amostra foi constituída por 59 pacientes, sendo que destes 33,9\% $(n=20)$ cursaram com delirium. As características clínicas da amostra estão descritas na tabela 01. Verifica-se que em relação ao diagnóstico da admissão, destaca-se o traumatismo crânio encefálico com $25,0 \%$ da amostra $(n=15)$ e que houve predominância de indivíduos do sexo masculino (57,6\%; n=34). A idade média dos pacientes foi de aproximadamente 60 anos. 
Tabela 1. Perfil clínico dos participantes. Salvador, Bahia, Brasil, 2019

\begin{tabular}{lcc}
\hline \multicolumn{1}{c}{ Variáveis } & $\mathbf{N}$ & \% \\
\hline Idade (Média \pm DP) & $59,5 \pm 2,1$ & \\
Dias de internamento na UTI (Média \pm DP) & $3,1 \pm 2,9$ & \\
SAPS 3 & $39,0 \pm 8,1$ & 42,4 \\
Sexo & 25 & 57,6 \\
Feminino & 34 & \\
Masculino & & 25,4 \\
Diagnóstico da admissão & 15 & 11,9 \\
Traumatismo Cranioencefálico & 7 & 10,2 \\
Queda & 6 & 10,2 \\
Doença renal crônica & 6 & 8,5 \\
Acidente vascular cerebral & 5 & 5,1 \\
Politrauma & 3 & 3,4 \\
Perfuração por arma de fogo & 2 & 3,4 \\
Traumatismo Raquimedular & 2 & 1,7 \\
Arritmia & 1 & 20,3 \\
Insuficiência respiratória aguda & 12 & \\
Outros & & \\
\hline DP: desvio-padrão
\end{tabular}

DP: desvio-padrão

Fonte: Elaborado pela autora

A tabela 02 apresenta o perfil do delirium na amostra. Entre os subtipos motores, o espectro mais frequente foi o hipoativo, com $50 \%$ dos casos $(n=10)$. Associadamente, observa-se que $90 \%$ dos pacientes apresentaram o início dos episódios nas primeiras 24 horas de internamento e a média de duração dos episódios foi de aproximadamente 02 dias.

Tabela 2. Perfil do delirium na amostra. Salvador, Bahia, Brasil, 2019

\begin{tabular}{lcc}
\hline \multicolumn{1}{c}{ Variáveis } & $\mathbf{N}$ & \% \\
\hline Espectros do delirium & & \\
Hipoativo & 10 & 50,0 \\
Hiperativo & 4 & 20,0 \\
Misto & 6 & 30,0 \\
Início dos episódios & & \\
Nas primeiras 24 horas de internamento & 18 & 90,0 \\
Após as 24 horas de internamento & 2 & 10,0 \\
Duração dos episódios em dias (Média \pm DP) & $1,9 \pm 1,2$ & \\
\hline
\end{tabular}

Fonte: Elaborada pela autora

Ao avaliar os fatores associados ao delirium (tabela 03), destaca-se que quase a totalidade das variáveis analisadas (exceto o alcoolismo) apresentaram uma maior frequência no grupo com delirium. Encontrou-se uma associação positiva e estatisticamente significante entre o uso de contenção física e ocorrência da disfunção (RR=3,10; IC95\% 1,30 - 7,43). Dos pacientes em delirium, 75\% utilizavam contenção física no momento da aplicação da CAM-ICU, assim como $60 \%$ desses pacientes estavam em uso de opioides. Não encontrou-se associação entre as variáveis transfusão sanguínea e alcoolismo e delirium. Quanto às variáveis uso de anticolinérgicos e sedativos, ventilação mecânica e hipertensão, estas apresentaram associação positiva com a ocorrência de delirium, contudo sem significância estatística. 
Tabela 3. Fatores associados à ocorrência de delirium em pacientes internados em Unidades de Terapia Intensiva. Salvador, Bahia, Brasil, 2019

\begin{tabular}{|c|c|c|c|c|}
\hline Variáveis investigadas & $\begin{array}{l}\text { Com delirium } \\
\qquad(\mathrm{N}=\mathbf{2 0})\end{array}$ & $\begin{array}{c}\text { Sem } \\
\text { delirium } \\
(\mathrm{N}=39)\end{array}$ & $\begin{array}{c}\text { Total } \\
(\mathrm{N}=59)\end{array}$ & $\begin{array}{c}\text { RR } \\
\text { (IC 95\%) } \\
\end{array}$ \\
\hline Anticolinérgicos & $4(20,0)$ & $3(7,7)$ & $7(11,9)$ & $\begin{array}{c}1,85 \\
(0,86-3,97)\end{array}$ \\
\hline Sedação & $3(15,0)$ & $5(12,8)$ & $8(13,6)$ & $\begin{array}{c}1,12 \\
(0,42-2,98)\end{array}$ \\
\hline Ventilação mecânica & $4(20,0)$ & $7(17,9)$ & $11(18,6)$ & $\begin{array}{c}1,09 \\
(0,45-2,62)\end{array}$ \\
\hline $\begin{array}{c}\text { Transfusão sanguínea } \\
\text { Alcoolismo }\end{array}$ & $4(20,0)$ & $8(20,5)$ & $12(20,3)$ & $\begin{array}{c}0,97 \\
(0,40-2,39)\end{array}$ \\
\hline Contenção física & $7(35,0)$ & $14(35,9)$ & $21(36,8)$ & $\begin{array}{c}1,00 \\
(0,46-2,14)\end{array}$ \\
\hline Hipertensão Arterial & $15(75,0)$ & $14(35,9)$ & $29(49,2)$ & $\begin{array}{c}3,10 \\
(1,30-7,43)\end{array}$ \\
\hline \multirow[t]{2}{*}{ Opióides } & $10(50,0)$ & $18(46,2)$ & $28(48,3)$ & $\begin{array}{c}1,07 \\
(0,52-2,17)\end{array}$ \\
\hline & $12(60,0)$ & $16(41,0)$ & $28(47,5)$ & $\begin{array}{c}1,66 \\
(0,79-3,46)\end{array}$ \\
\hline
\end{tabular}

RR - Risco Relativo; IC - Intervalo de Confiança. Valores apresentados em n (\%).

Fonte: Elaborada pela autora

\section{Discussão}

Através deste estudo, foram avaliados a incidência e os fatores clínicos associados ao delirium. Sabe-se que as análises sobre a incidência de delirium na UTI podem variar de acordo com as características da população que esteja sendo estudada e com o método de detecção utilizado. A incidência de delirium encontrada nesta pesquisa foi de aproximadamente $34 \%$, dado semelhante ao encontrado em uma meta-análise realizada com 42 estudos, onde o estado confusional agudo incidiu em 5.280 pessoas entre os 16.595 pacientes pesquisados $(31,8 \%)^{4}$.

Em relação ao perfil clínico dos pacientes em delirium, o espectro hipoativo foi o mais incidente no presente estudo, seguido do misto. Segundo meta-análise realizada por canadenses em 2018, onde os mesmos utilizaram incidência combinada, observou-se que entre os pacientes que apresentaram delirium houve uma maior incidência do subtipo hipoativo (11\%), seguido do misto $(7 \%)$ e por último o hiperativo $(4 \%)^{2}$, resulta- dos que corroboram com os achados desse estudo. Associadamente, outros autores reiteram que o subtipo hipoativo encontra-se entre os mais prevalentes, chegando até a $80 \%$ dos casos, seguido do subtipo misto $^{10}$.

Diante desses achados, ressalta-se a importância da utilização de escalas validadas para o reconhecimento dos subtipos e diagnóstico precoce do delirium. As recomendadas para a utilização em pacientes críticos já foram validadas para o português através do estudo de Gusmão-Flores e colaboradores ${ }^{11}$. Segundo estudo recente que objetivou analisar as propriedades psicométricas de ferramentas de avaliação de delirium, as do CAM-ICU e o ICDSC foram consideradasmuito boas quando comparadas às outras escalas disponíveis (19.6 e 19.2, respectivamente), constituindo-se nos instrumentos mais válidos e confiáveis para aplicação em pacientes críticos $^{12}$. Ratifica-se então o uso das mesmas, já que são de fácil e rápida aplicação (3 a 5 minutos) e proporcionam diagnóstico acurado da disfunção. 
Dados desta pesquisa demonstram que o delirium se instalou nas primeiras 24 horas de internamento em 90\% dos participantes, diferente do evidenciado em outros estudos que encontraram mediana de tempo para início dos episódios de 3,5 dias ${ }^{13}$. Sabe-se que existem escores de risco para identificar pacientes com risco de desenvolver delirium. Nas unidades de terapia intensiva, têm-se os escores Early Prediction Model for Delirium in ICU Patients (E-PRE-DELIRIC), que avalia 09 fatores de risco para o delirium logo na admissão do paciente na unidade crítica e o Delirium Prediction Model for Intensive Care Patients (PREDELIRIC) ${ }^{14}$, que avalia 10 fatores de risco para o delirium facilmente identificáveis nas primeiras 24 horas de internação, ambos apresentando elevado valor preditivo. Através do cálculo desses escores de risco, é possível avaliar o risco individual de cada paciente, tendo possibilidade de iniciar precocemente as medidas preventivas para os pacientes com moderado e alto risco de delirium, evitando assim à sua ocorrência, aumento dos custos hospitalares e desfechos clínicos desfavoráveis.

Estudos mostram que os fatores de risco para delirium podem ser predisponentes ou precipitantes. Sabese que vários dos fatores precipitantes (conhecidos como modificáveis) podem ser minimizados. Dentre estes estão a diminuição da sedação e do tempo de ventilação mecânica, mobilização precoce, correção de distúrbios hidroeletrolíticos e hipóxia, remoção de dispositivos invasivos, entre outros ${ }^{15}$. Outras medidas preventivas que foram testadas para a diminuição da ocorrência de delirium através de um estudo prospectivo realizado em um Hospital Universitário nos EUA foram redução da privação de sono, o uso de aparelhos auditivos e óculos. Nos resultados do estudo mostra-se que houve redução significativa dos quadros de delirium, tendo impacto também na duração dos episódios ${ }^{16}$.

Quanto à duração dos episódios de delirium em nossa pesquisa, os resultados foram similares aos de um estudo multicêntrico realizado em dezesseis UTIs norte-americanas com 420 participantes em que encontraram uma mediana de dois dias ${ }^{13}$. Porém outra pesquisa realizada explana que o delirium pode durar persistentemente até 10 dias seguidos na UTI ${ }^{12}$.
Em relação ao perfil clínico dos participantes, verificou-se que a maior porcentagem do estudo foi composta por pacientes do sexo masculino $(57,4 \%)$ e com idade média de aproximadamente 60 anos, o que é corroborado por pesquisa realizada em uma UTI em São Paulo, que demonstra que dos $46,3 \%$ dos pacientes que apresentaram delirium, 60,9\% foram do sexo masculino com idade média de aproximadamente 65 anos $^{17}$. Constatou-se através de pesquisa que o delirium é mais frequente em idosos, chegando a acometer até $72 \%$ destes, quando internados em UTI. Tal fato pode ser atribuído aos idosos apresentarem vários fatores de risco que são comprovadamente associados ao delirium ${ }^{18}$.

Em relação aos diagnósticos médicos encontrados em nosso estudo, percebe-se que houve uma alta incidência de pacientes com Traumatismo Crânio Encefálico (TCE), seguidos de queda, fato que pode ser justificado pela pesquisa ter sido realizada em um Hospital Referência em Trauma. De acordo com um estudo de coorte realizado com 140 pacientes adultos e idosos de um hospital na Paraíba, não foi encontrada associação estatisticamente significante entre o TCE e a ocorrência de delirium ${ }^{19}$. Contudo, atuais diretrizes apresentam o trauma como fator de risco fortemente associado à ocorrência de delirium ${ }^{7}$.

Ao avaliarmos as variáveis clínicas associadas à ocorrência de delirium, o uso de restrição física mostrou-se como a única variável investigada que apresentou associação positiva e estatisticamente significante, elevando em 03 vezes o risco de ocorrência desta disfunção. Em pesquisa de coorte realizada em uma UTI de um hospital em São Paulo, identificou-se que o uso da restrição física pode aumentar em 44,3 vezes a chance de desenvolver delirium ${ }^{17}$. De acordo com pesquisa realizada, há um uso indiscriminado de contenções mecânicas nas Unidades de Terapia Intensiva. Fato que se justifica devido ao paciente nesses ambientes estarem em uso de inúmeros dispositivos que não podem ser exteriorizados. Outro momento comum para aplicação da restrição é nos pacientes com delirium hiperativo. Tal procedimento é realizado para evitar eventos adversos como quedas e evasão. Porém, sabe-se que tanto aplicação quanto a manutenção da contenção física devem ser avaliadas de forma criteriosa pela equipe enfermagem, pois muitas vezes são utilizadas inadvertidamente ou são mantidas por períodos duradouros sem indicação, contribuindo assim para a ocorrência do delirium. 
Outra variável que teve destaque nos resultados do estudo foi o uso de opioides. Dentre os pacientes que apresentaram delirium, $60 \%$ destes utilizaram esta droga como analgésico, o que é comum dentre pacientes de unidades de trauma visto os quadros de algia intensa, porém não houve significância estatística. Contudo, pesquisas informam que o uso da morfina pode aumentar em três vezes o risco de desenvolver delirium ${ }^{17}$.

As variáveis sedação, anticolinérgicos, hipertensão e ventilação mecânica também apresentaram associação positiva com a disfunção, porém sem significância estatística. Pesquisas afirmam que os sedativos são amplamente utilizados em ambientes de terapia intensiva e especialmente os benzodiazepínicos podem causar complicações importantes nos pacientes, como aumento do tempo de ventilação mecânica, aumento da incidência de delirium e consequentemente maior mortalidade ${ }^{5,17,20}$.

Aponta-se em pesquisa efetuada em Escola de Medicina de Universidade de Nashville nos EUA, que dos pacientes submetidos à ventilação mecânica, 83,3\% apresentaram delirium ${ }^{8}$. Em estudo publicado recentemente, dos 420 participantes em ventilação mecânica, 53,8\% apresentaram CAM positivo ${ }^{13}$. Em nosso estudo, $20 \%$ dos pacientes em delirium encontravam-se mecanicamente ventilados.

Segundo o Clinical practice guidelines for the management of pain, agitation, and delirium in adult patients in the intensive care unit, publicado em 2013, a hipertensão está dentre os quatro fatores fortemente associados à ocorrência desse estado confusional agudo no paciente internado em UTI5. No estudo em tela, observou-se que dos pacientes em delirium, 50\% eram hipertensos. Outro fator que é altamente vinculado ao desenvolvimento desta disfunção é o uso de alguns fármacos, como por exemplo, os anticolinérgicos e benzodiazepínicos 5 . Dos pacientes com delirium que compuseram a nossa amostra, 20\% estavam em uso de anticolinérgicos. Segundo os dados apresentados neste estudo, estar em uso deste fármaco aumenta em aproximadamente duas vezes o risco de desenvolver essa disfunção cerebral, porém sem significância estatística.

\section{Conclusão}

Os resultados desse estudo revelaram uma incidência de delirium elevada, contudo semelhante ao encontrado em outras produções científicas, com predomínio do espectro hipoativo. Entre as variáveis investigadas, houve uma associação positiva e estatisticamente significante entre uso de restrição física e a ocorrência de delirium nos participantes da pesquisa. Tal achado reitera a importância da indicação criteriosa do uso da contenção mecânica e da avaliação diária da necessidade de sua manutenção, visto que é uma medida facilmente gerenciável.

Como limitações desse estudo têm-se a amostra não probabilística de conveniência, o que compromete a generalização dos resultados. Outro fator limitante refere-se à aplicação da escala CAM-ICU ter sido realizada somente uma vez ao dia, podendo ter levado ao subdiagnóstico de delirium na amostra da pesquisa.

Espera-se com os resultados desse estudo contribuir com o meio científico e alertar os profissionais de saúde sobre esta disfunção, que ainda nos dias atuais mostra-se tão invisível. Ressalta-se então a necessidade de atualização profissional para conhecimento dos métodos de monitorização do delirium e a necessidade de implementação precoce de medidas preventivas.

\section{Contribuições dos autores}

Oliveira MTS participou da coleta de dados da pesquisa. Pessoa LSC participou da coleta de dados da pesquisa e construção do banco de dados. Silva DS realizou a análise estatística e construção da metodologia. Mercês MC realizou a análise estatística e interpretação dos resultados. Lago MS participou da concepção, delineamento, coleta de dados, redação do artigo científico e interpretação dos resultados. Faustino TN participou da concepção, delineamento, interpretação dos resultados e revisão da redação do artigo científico.

\section{Conflitos de interesses}

Nenhum conflito financeiro, legal ou político envolvendo terceiros (governo, empresas e fundações privadas, etc.) foi declarado para nenhum aspecto do trabalho submetido (incluindo, mas não se limitando a subvenções e financiamentos, participação em conselho consultivo, desenho de estudo, preparação de manuscrito, análise estatística, etc.). 


\section{Referências}

1. American Psychiatric Association. Manual diagnóstico e estatístico de transtornos mentais: DSM-5. Porto Alegre: Artmed; 2014.

2. Krewulak KD, Stelfox HT, Leigh JP, Ely EW, Fiest KM. Incidence and Prevalence of Delirium Subtypes in an Adult ICU: A Systematic Review and Meta-Analysis. Crit Care Med. 2018;46(12):2029-35. doi: $10.1097 / C C M .0000000000003402$

3. Wolters AE, Peelen LM, Veldhuijzen DS, Zaal IJ, Lange DW, Pasma W et al. Long-Term Self-Reported Cognitive Problems After Delirium in the Intensive Care Unit and the Effect of Systemic Inflammation. J Am Geriatr Soc. 2017;65(4):786-91. doi: 10.1111/ jgs. 14660

4. Salluh JIF, Wang H, Schneider EB, Nagaraja N, Yenokyan G, Damluji $A$ et al. Outcome of delirium in critically ill patients: systematic review and meta-analysis. BMJ. 2015;350:h2538. doi: 10.1136/bmj.h2538

5. Barr J, Fraser GL, Puntillo K, Ely EW, Gélinas C, Dasta JF et al. Clinical practice guidelines for the management of pain, agitation, and delirium in adult patients in the intensive care unit. Crit Care Med. 2013;41(1):263-306. doi: 10.1097/CCM.0b013e3182783b72

6. Park SY, Lee HB. Prevention and management of delirium in critically ill adult patients in the intensive care unit: a review based on the 2018 PADIS guidelines. Acute Crit Care. 2019;34(2):117-125. doi: $10.4266 /$ acc. 2019.00451

7. Devlin JW, Skrobik Y, Gélinas C, Needham DM, Slooter AJC, Pandharipande PP et al. Clinical Practice Guidelines for the Prevention and Management of Pain, Agitation/Sedation, Delirium, Immobility, and Sleep Disruption in Adult Patients in the ICU. Crit Care Med. 2018;46(9):e825-e873. doi: 10.1097/ CCM.0000000000003299

8. Ely EW, Margolin R, Francis J, May L, Truman B, Dittus R et al. Evaluation of delirium in critically ill patients: validation of the Confusion Assessment Method for the Intensive Care Unit (CAMICU). Crit Care Med. 2001;29(7):1370-9. doi: 10.1097/00003246200107000-00012

9. Brasil. Conselho Nacional de Saúde. Resolução 466/12. Trata de pesquisas em seres humanos e atualiza a resolução 196. Diário Oficial da União. 2012.

10.Stagno D, Gibson C, Breitbart W. The delirium subtypes: a review of prevalence, phenomenology, pathophysiology, and treatment response. Palliat Support Care. 2004; 2(2):171-9. doi: $10.1017 / \mathrm{s} 1478951504040234$

11.Gusmao-Flores D, Salluh JI, Dal-Pizzol F, Ritter C, Tomasi CD, Lima MA et al. The validity and reliability of the Portuguese versions of three tools used to diagnose delirium in critically ill patients. Clinics. 2011;66(11):1917-22. doi: 10.1590/S180759322011001100011
12. Gélinas C, Bérubé M, Chevrier A, Pun BT, Ely EW, Skrobik Y et al. Delirium Assessment Tools for Use in Critically III Adults: A Psychometric Analysis and Systematic Review. Crit Care Nurse. 2018;38(1):38-49. doi: $10.4037 / \mathrm{ccn} 2018633$

13. Mehta S, Cook D, Devlin JW, Skrobik Y, Meade M, Fergusson $D$ et al. Prevalence, risk factors, and outcomes of delirium in mechanically ventilated adults. Crit Care Med. 2015;43(3):557-66. doi: $10.1097 / C C M .0000000000000727$

14. Wassenaar A, Boogaard M, Achterberg T, Slooter AJ, Kuiper MA, Hoogendoorn ME et al. Multinational development and validation of an early prediction model for delirium in ICU patients. 2015;41(6):1048-56. doi: 10.1007/s00134-015-3777-2

15. Patel RP, Gambrell M, Speroff T, Scott TA, Pun BT, Okahashi J et al. Delirium and sedation in the intensive care unit: survey of behaviors and attitudes of 1384 healthcare professionals. Crit Care Med. 2009;37(3):825-32. doi: 10.1097/ CCM.0b013e31819b8608

16. Inouye SK, Bogardus Jr ST, Charpentier PA, Leo-Summers L, Acampora D, Holford TR et al. A multicomponent intervention to prevent delirium in hospitalized older patients. N Engl J Med. 1999;340(9):669-76. doi: 10.1056/NEJM199903043400901

17. Mori S, Takeda JRT, Carrara FSA, CR Cohrs, Zanei SSV, Whitaker IY. Incidence and factors related to delirium in an Intensive Care Unit. Rev Esc Enferm USP. 2016;50 (4):587-93. doi: 10.1590/S0080623420160000500007

18. Pisani MA, Kong SYJ, KasI SV, Murphy TE, Araujo KLB, Van Ness $\mathrm{PH}$. Days of delirium are associated with 1-year mortality in an older intensive care unit population. Am J Respir Crit Care Med. 2009;180(11):1092-7. doi: 10.1164/rccm.200904-05370C

19. Muñoz RLS, Tagushi LC, Martins GCCS, Andrade MR, Lima KMSR. Prevalence and factors associated with delirium occurrence in hospitalized adults. Rev Soc Bras Clin Med. 2012;10(4): 285-90.

20. Wiatrowski R, Norton C, Giffen, D. Analgosedation: Improving Patient Outcomes in ICU Sedation and Pain Management. Pain Manag Nurs. 2016; 17(3):204-17. doi: 10.1016/j.pmn.2016.02.052 